Article DOI : https://doi.org/10.35219/ann-ugal-math-phys-mec.2019.1.05

\title{
EDGES IDENTIFICATION BASED ON THE DERIVATIVE FILTERS AND FRACTAL DIMENSION
}

\author{
Luminița Moraru ${ }^{1}$, Simona Moldoveanu ${ }^{2}$, Lenuța Pană ${ }^{1}$ \\ ${ }^{1}$ Faculty of Science and Environment, "Dunărea de Jos" University of Galați, 47 Domnească Street, RO- \\ 800008, Galați, Romania \\ 2 Faculty of Automation, Computers Sciences, Electronics and Electrical Engineering , "Dunărea de Jos" \\ University of Galați ,2 Stiinței Street, RO-800210, Galați, Romania
}

\begin{abstract}
The purpose of this paper is to offer a comparative study on the fractal dimension (D) used to differentiate edges in brain images processed using first-order derivative filters (Prewitt, Roberts) and second-order derivative filters (Laplacian and Laplacian of Gaussian). PDw (proton density) and $\mathrm{T} 2 \mathrm{w}$ (T2-weighted type) brain images of healthy patients and patients diagnosed with metastatic bronchogenic carcinoma (MBC) are used. Experimental results showed that secondorder derivative filters clearly separate healthy controls from diseased patients while the first-order derivative filters create false edges that affect the fractal dimension (D) values. The KullbackLeibler divergence (DKL) determines that the probability distribution of the "real" fractal measurements, specific to healthy patients is different from the probability distribution of the "arbitrary" fractal dimensions, specific to patients with MBC. The highest value to the distance DKL is for Prewitt filter. The value of distance DKL is close to zero for Laplacian, LoG and Roberts filters.
\end{abstract}

Keywords: edges, first and second-order derivative filters, fractal dimension, Kullback-Leibler divergence. (3-5 keywords, TNR 10 pt.)

\section{INTRODUCTION}

Edge detection is an important stage for various digital image processing techniques. It is largely used in medical images processing and analyzing [1,2]. Edge detection is important in locating discontinuities and significant transitions in gray level values associated to the pixels in the image $[1,3]$. Detection of edges in an image is performed by analyzing the pixels distribution and evaluating their intensities around a given, fixed pixel. Sharp variations between two neighborhood regions allow to a pixel to be classified as belonging to an edge $[1,5]$.

Edge detection using spatial filtering has led to improved methods for content classification by detecting boundaries between image regions, the shapes and locations of different objects [1]. Each filter is characterized by its own mask or window, i.e. a specific matrix of kernel coefficients (weights) or specific operations and with a defined size. It convolutes the image and produces various discrete approximations with the final goal to emphasize edges [5]. In the resulted edge-map, the pixels belonging to an edge are determined by examining the relationship between certain pixels their neighbors [1,5]. applied:

To detect edges in the cerebral tissues of brain images, the following filters were sequentially 
Roberts and Prewitt first-order derivative filters, who used first order approximations of the gradient operator;

Laplacian and Laplacian of Gaussian second-order derivative filters, who used second order approximations of the gradient operator

Prewitt and Roberts operators use the first derivative of the image function to describe the position of an image pixel (x, y) and the gray value $\mathrm{A}(\mathrm{x}, \mathrm{y})$ of the pixel at coordinates $(\mathrm{x}, \mathrm{y})$ [5].

The Laplacian operator uses the second order derivative function to describe the position of an image pixel A (x, y) and the gray value of $(\mathrm{x}, \mathrm{y})$ at coordinates $(\mathrm{x}, \mathrm{y})$. It finds the true edges, and tests the wider areas around the pixel, but fails when the image contains corners and curves.

The LoG operator uses the following three successive operations: filtering using a Gaussian filter; enhancement using a Laplacian operator (the second derivative); and, edge detection as or a zero crossing in the second derivative.

The LOG filter compensates the variation of pixel intensities values occurring when different image sources with the same content are investigated. Additionally, LoG provides sharpened the final results.

The fractal dimension of the edge maps provided by four filters correlated to the KullbackLeibler divergence are used to check the accuracy of the filtering process.

The fractal geometry is based on chaos theory and was proposed by Mandelbrot [7]. It facilitates the understanding and description of complex biological systems that is beyond of a simply Euclidean geometry analysis [6]. In 1975, Benoit Mandelbrot described fractals as geometric objects or dynamic constructs that share a degree of similarity to the original object in a wide range of scales [7].

Majumdar et al. 1988 [8] uses axial, coronal and sagittal images of the brain to compute the fractal dimension of the surface bounded by the grey matter. The fractal dimension was used for a quantitative characterization of the area bordering the grey matter and as a feature for monitoring and identification of anomalies and changes in the brain development. By analyzing the histogram of the median values of the fractal dimensions they reported a fractal dimension of 2.6 as the most frequent value, for a normal brain.

Esteban et al. [9] compared T1w and T2w images of healthy patients and of different patients showing multiple sclerosis lesion. The fractal dimension was used to determine anomalies of the grey substance. The authors have shown that fractal dimension has high values for the grey matter of patients with multiple sclerosis, as a result of the morphological complexity of brain tissue. Higher fractal dimensions were obtained to patients with early stages of multiple sclerosis.

Jayasuriya et al. [10] reported a novel approach able to identify the symmetry in the cerebral structures using the mid-sagittal plan, for 3D MRI images. They used concepts of fractal dimension and lacunarity. The proposed algorithm has some limitations because it requires a high degree of symmetry between brain hemispheres. For images showing severe asymmetries due to tumors or neurological injuries the accuracy of the mid-sagittal plan position can be biased.

Hoyos et al. [11] analyzed T1w MR images containing primary tumors. The in vivo evolution of 3D tumors was analyzed based on the fractal dimension values and local roughness of the cerebral tissue. They have shown that as the lesion becomes more aggressive, the complexity of the host tumor interface complexity increases.

In this study, the fractal dimension (D) computed in the framework of the box-counting method for binary images produced using first order and second order filters [12, 13].

To estimate the distribution of the fractal dimensions, the mean and standard deviation were computed. To compare two distributions, i.e. the "real" probability distribution attributed to healthy (H) patients and the "arbitrary" distribution attributed to patients with MBC, all images were analyzed using the Kullback-Leibler divergence (DKL). 


\section{METHODS}

\subsection{Mathematical approaches}

a. The first-order derivative filters and the second-order derivative filters

An image is defined by an image function $A(x, y)$ that gives the intensity of the gray levels at pixel position $(\mathrm{x}, \mathrm{y})$. The gradient vector of the image function, is defined as [16]:

$$
\nabla A(\boldsymbol{x}, \boldsymbol{y})=\left[G_{x} G_{y}\right]=\left[\frac{\partial A(\boldsymbol{x}, \boldsymbol{y})}{\partial x} \frac{\partial A(\boldsymbol{x}, \boldsymbol{y})}{\partial y}\right]
$$

\section{a.1. Prewitt Operator}

The Prewitt filter is used to detect vertical and horizontal directions of the edges of an image by locating those pixel values defined by steep gray values [15]. The Prewitt operator consists of two $3 \mathrm{x}$ 3 convolution masks, $[14,16]$ :

$$
\begin{gathered}
G_{y}=\left[\begin{array}{ccc}
+1 & 0 & -1 \\
+1 & 0 & -1 \\
+1 & 0 & -1
\end{array}\right] * A(\boldsymbol{x}, \boldsymbol{y}) \\
G_{x}=\left[\begin{array}{ccc}
+1 & +1 & +1 \\
0 & 0 & 0 \\
-1 & -1 & -1
\end{array}\right] * A(\boldsymbol{x}, \boldsymbol{y})
\end{gathered}
$$

where $\mathrm{A}$ is the image source and $*$ is the 2-dimensional convolution operation

\section{a.2. Roberts Operator}

Roberts operator consists of two $3 \times 3$ convolution masks, and estimates directional gradient along two image diagonals [3]:

$$
\begin{aligned}
G_{x} & =\left[\begin{array}{ccc}
0 & 0 & 0 \\
0 & -1 & 0 \\
0 & 0 & 1
\end{array}\right] * A(x, y) \\
G_{y} & =\left[\begin{array}{ccc}
0 & 0 & 0 \\
0 & 0 & -1 \\
0 & 1 & 0
\end{array}\right] * A(x, y)
\end{aligned}
$$

\section{a.3. The Laplacian operator}

The Laplace operator is computed using the second order derivative approximations of the function A $(x, y)$. This operator is noise sensitive so it is often combined with a Gaussian filter to decrease sensitivity to noise [3].

The Laplacian filter searches the zero crossing points of the second order derivatives of the image function $[16,17]$.

$$
\nabla^{2} A(\boldsymbol{x}, \boldsymbol{y})=\frac{\partial^{2} A(\boldsymbol{x}, \boldsymbol{y})}{\partial x^{2}}+\frac{\partial^{2} A(\boldsymbol{x}, \boldsymbol{y})}{\partial y^{2}}
$$

It determines those rapid changes in adjacent pixel values that belong to an edge or to a continuous progression. The output image contains gray pixels of different intensity values. The zero value 
indicates the areas of constant intensity whereas those areas showing values $<0$ or $>0$ are placed in the vicinity of an edge.

\section{a.4. The Laplacian of Gaussian (LoG) operator}

combines the Laplacian and Gaussian functions to obtain a new operator LoG is $\mathrm{h}$ (x,y) define by [18]

$$
\begin{aligned}
g(\boldsymbol{x}, \boldsymbol{y}) & =-\frac{1}{2 \pi s^{2}} \exp \left[-\frac{x^{2}+y^{2}}{2 s^{2}}\right] \\
h(\boldsymbol{x}, \boldsymbol{y}) & : \nabla^{2}[g(\boldsymbol{x}, \boldsymbol{y}) * f(\boldsymbol{x}, \boldsymbol{y})]=\left[\nabla^{2} g(\boldsymbol{x}, \boldsymbol{y})\right] * f(\boldsymbol{x}, \boldsymbol{y})=h(\boldsymbol{x}, \boldsymbol{y})^{*} f(\boldsymbol{x}, \boldsymbol{y}) \\
\nabla^{2} g(\boldsymbol{x}, \boldsymbol{y}) & =\frac{x^{2}+y^{2}-2 s^{2}}{2 \pi s^{4}} \exp \left[-\frac{x^{2}+y^{2}}{2 s^{2}}\right]
\end{aligned}
$$

where

$\mathrm{g}(\mathrm{x}, \mathrm{y})$ is the Gaussian kernel, $\mathrm{f}(\mathrm{x}, \mathrm{y})$ is the image source, $\mathrm{s}$ is the standard deviation of a Gaussian distribution.

The Gaussian operator suppresses the noise before using Laplace operator for edge detection. LoG detects areas in which intensity changes rapidly, namely the function's values are positive on the darker side (pixel values close to zero) and negative on the brighter side (pixel values close to 255). In practice, a threshold value is also used to determine the zero crossing that select the most pronounced points of the edge (defined as the difference between the maximum positive and minimum negative values).

\section{b. The box-counting method}

The irregular or convoluted objects such as cerebral surfaces are more accurate characterized using the fractal geometry. In this case, the Euclidean geometry that is associated to regular shapes or surfaces is not a proper choice and a non-Euclidean descriptor of the morphology structure called the fractal dimension D is used. An object of size D can be describe using objects that are self similar, over a range of scales, so that it can be divided into $\mathrm{nD}$ objects " $\mathrm{n}$ " times smaller. The box-counting method is a tool to determine the fractal dimension. It is an iterative method; at each step it counts the number of self similar elements that divide the image.

The number of elements is denoted by $\mathrm{N}$, the size of the object by $\mathrm{r}$, so that, the fractal dimension $\mathrm{D}$ is defined as follows [19]:

$$
D=\frac{\log N(r)}{\log \frac{1}{r}}
$$

This process continues until the smallest item contains the smallest irregularity in the image. Note that $\mathrm{D}$ is not always an integer.

\section{c. The Standard Deviation}

The standard deviation (DS) indicates how accurate the mean of the fractal dimension xi (in our case) is likely to be compared to the true population mean $\bar{x}$, computed for a given image [21].

$$
D S=\sqrt{\frac{\sum_{i=1}^{n}\left(x_{i}-\bar{x}\right)^{2}}{(n-1)}}
$$

where " $n$ " is the number of MRI images

\section{d. The Kullback Leibler divergence}

The Kullback -Leibler divergence (DKL) or the relative entropy, is a quasi-metric which estimates how the "real" fractal measurements, specific to healthy patients is different from the 
probability distribution of the "arbitrary" fractal dimensions, specific to patients with MBC [13]. DKL is computed as [20]

$$
D_{K L}(H \| M B C)=F D_{H} \log \frac{F D_{M B C}}{F D_{H}}
$$

where, FDH is the "real" fractal measurements, specific to healthy patients $(\mathrm{H})$ patients and the FDMC is the "arbitrary" fractal dimensions, specific to patients with MBC. If the metric values are close to zero, the two distributions are almost similar [14].

\subsection{Subjects, image acquisition and post-processing}

The numerical computing environment is the MATLAB R2017a software and Image Processing toolbox. The hardware used for experiments was a computer with the following specifications: CPU Inter (R) Core (TM) i7-8550U @ 1.80 GHz CPU; memory (RAM) 8 GB DDR4; video card GeForce MX150 4 GB; hard disk 256 GB SSD, Windows 10, 64-byte.

The image dataset includes $60 \mathrm{PDw}$ and T2w magnetic resonance images for healthy $(\mathrm{H})$ patients and MBC patients. The images were downloaded from Harvard's Whole Brain Atlas website for free.

\subsection{Flow chart}

The flow chart for post-processing and analysis is shown in Fig.1

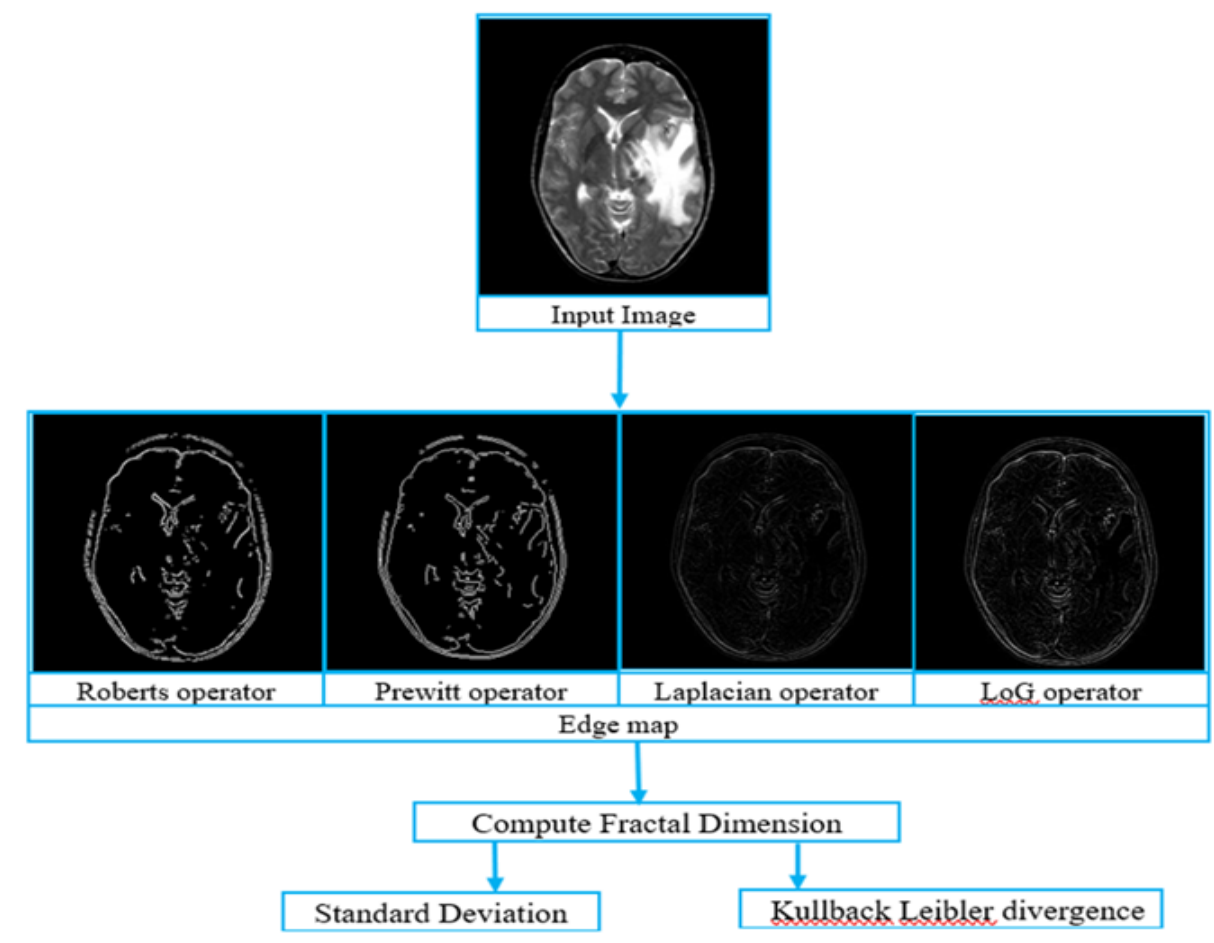

Fig. 1 Flow chart

\section{RESULTS}

Fractal dimension values in image edge map generation for $30 \mathrm{PDw}$ and $\mathrm{T} 2 \mathrm{w}$ images for $\mathrm{H}$ patients and $30 \mathrm{PDw}$ and $\mathrm{T} 2 \mathrm{w}$ images for the MBC patients are shown in Fig. 2, for different filtering operations. Table 1 summarizes the results of standard deviations of the fractal dimension 
in binary edge maps, for both patient groups. Table 2 presents the DKL values associated to fractal dimension in the edge maps.

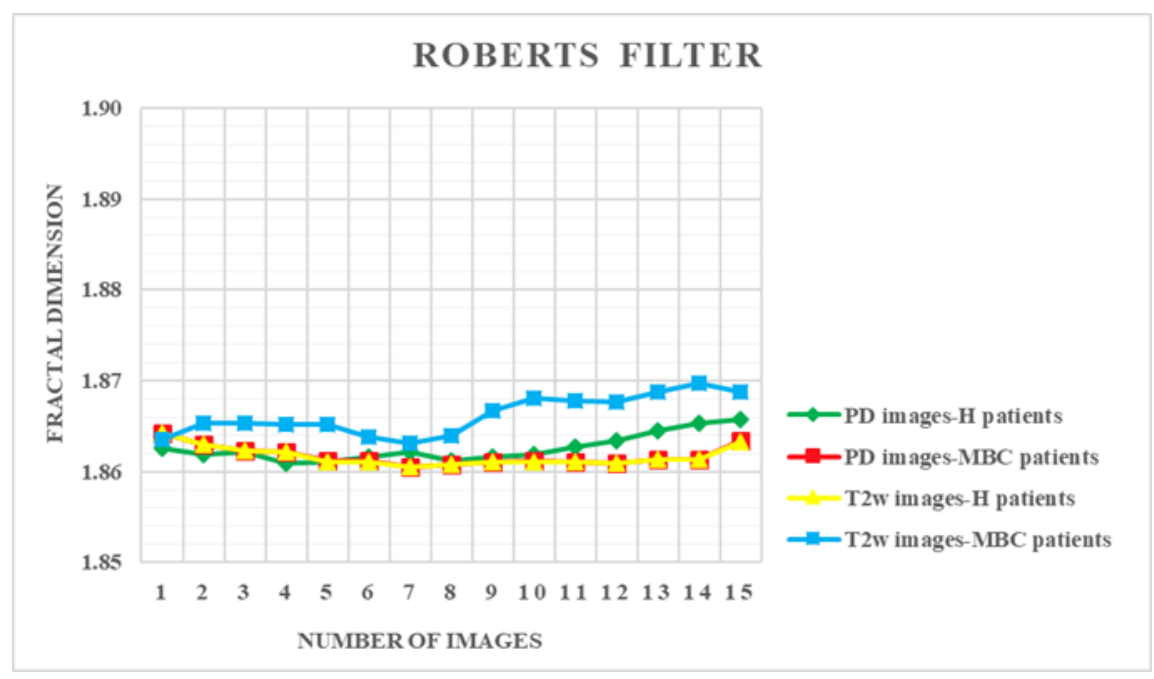

a)

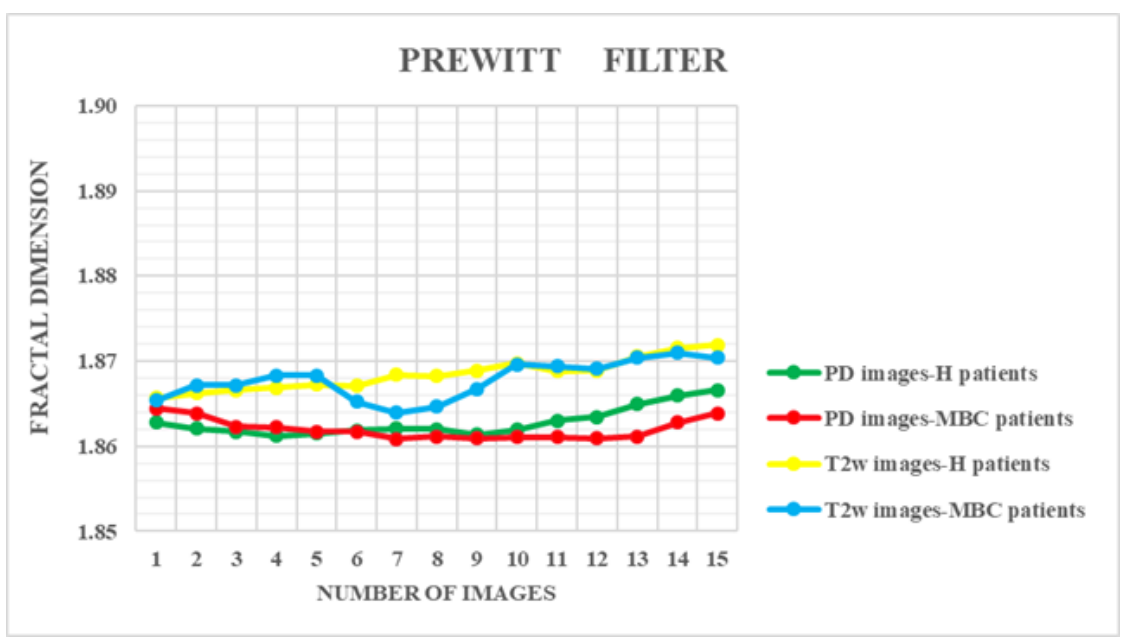

b)

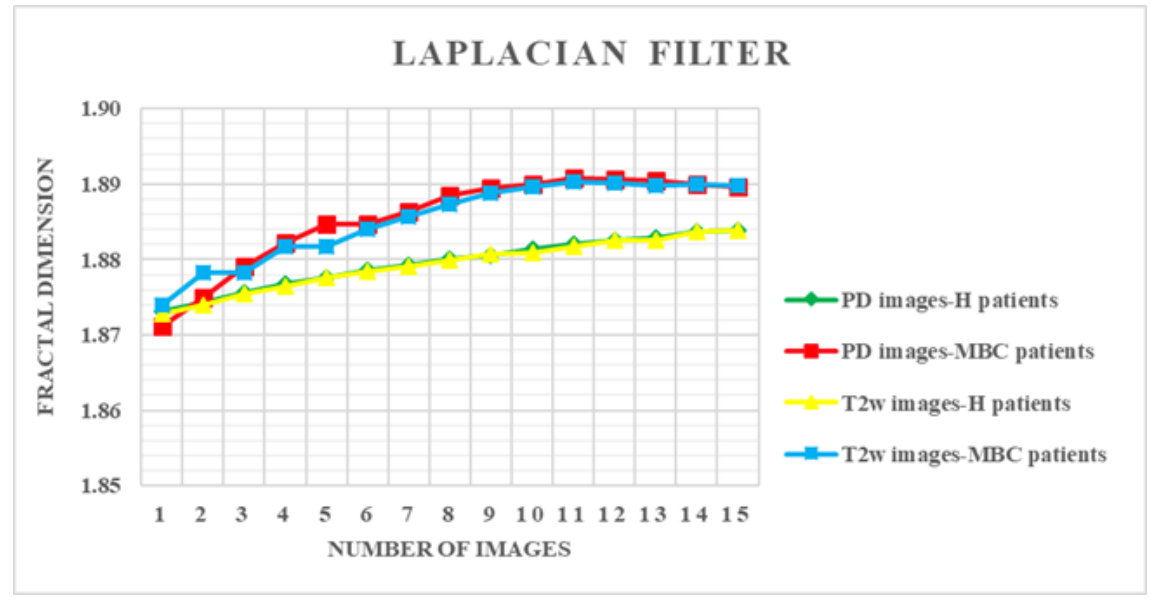

c) 


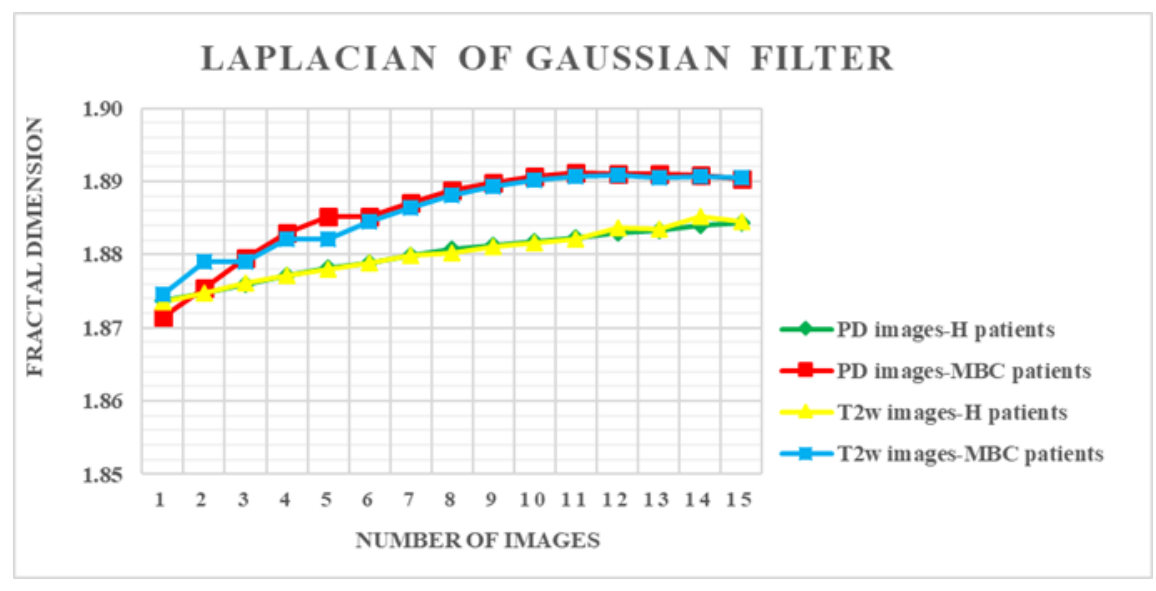

d)

Fig.2 Fractal dimension values in image edge maps generated for PDw and T2w images:

(a) the Roberts Filter; (b) the Prewitt Filter; (c) the Laplacian Filter; (d) the LoG Filter;

An analysis of the distribution of fractal dimensions values over the analyzed images leads to the following observations:

- images processed by using the Roberts and Prewitt operators (fig. 2a, 2b) show different fractal dimension values of the edge maps for both healthy and MBC patients, and for both image types ( $\mathrm{PDw}$ and $\mathrm{T} 2 \mathrm{w}$ ). It is one exception, when the fractal dimension values of $\mathrm{T} 2 \mathrm{w}$ image of healthy patients and PDw of MBC patients overlap. These two operators show a reduced effectiveness in separating the types of patients;

- for images filtered with the Lapacian and LoG (fig. 2 c, 2d), the fractal dimension values computed for healthy patients for both types of images are lower than those computed for patients with MBC. A higher fractal dimension value indicates a higher dissimilarity in the cerebral tissues, and have proved the effectiveness in differentiation between $\mathrm{H}$ and $\mathrm{MBC}$ patients.

Table 1. Mean \pm standard deviation of the fractal dimension in binary edge maps

\begin{tabular}{|l|l|l|l|l|}
\hline Operator & Roberts & Prewitt & Laplacian & Log \\
\hline PDw images-H patients & $1.863 \pm 0.002$ & $1.862 \pm 0.002$ & $1.879 \pm 0.003$ & $1.879 \pm 0.007$ \\
\hline $\begin{array}{l}\text { PDw images }- \text { MBC } \\
\text { patients }\end{array}$ & $1.862 \pm 0.001$ & $1.862 \pm 0.001$ & $\mathbf{1 . 8 8 5} \pm \mathbf{0 . 0 0 6}$ & $\mathbf{1 . 8 8 0} \pm \mathbf{0 . 0 1 3}$ \\
\hline T2w images-H patients & $1.862 \pm 0.001$ & $1.868 \pm 0.002$ & $1.879 \pm 0.003$ & $1.879 \pm 0.007$ \\
\hline $\begin{array}{l}\text { T2w images-MBC } \\
\text { patients }\end{array}$ & $1.866 \pm 0.002$ & $1.867 \pm 0.002$ & $\mathbf{1 . 8 8 5} \pm \mathbf{0 . 0 0 5}$ & $\mathbf{1 . 8 8 2} \pm \mathbf{0 . 0 1 1}$ \\
\hline
\end{tabular}

The data in Table 1 indicates how accurate is the mean of fractal dimensions of the MBC patients compared to the mean values of the healthy patients. The standard error increases for edge maps obtained by using the second order derivative operators for MBC patients, i.e. the means of D are more spread out, and this can indicate the pathological condition of the brain.

Table 2. DKL values associated to fractal dimension in the edge maps 


\begin{tabular}{|c|c|c|c|c|}
\hline DKL & Prewitt & Roberts & Laplacian Filter & LoG \\
\hline PDw Images & 7.608 & 0.005 & 0.039 & 0.039 \\
\hline T2w Images & 0.005 & 0.029 & 0.038 & 0.038 \\
\hline
\end{tabular}

Table 2 displays the DKL values computed by assigning the "real" fractal measurements, specific to healthy patients and "arbitrary" fractal dimensions, specific to patients with MBC. The highest DKL value is attributed to images processed with the Prewitt operator. At this stage, we can conclude that the Prewitt filter is not a good choice as an edge detector for declared purposes. As regards the Laplacian, LoG, and Roberts filters, the DKL values are very small. Moreover, DKL values are almost the same for the second-order derivative filters. This indicates a high fractal similarity of the edge maps provided by these operators. Correlated to the fractal dimension values in Table 1, the second-order derivatives filters allow for data distributions of MBC patients that differ significantly from the distribution specific of the $\mathrm{H}$ patients.

\section{CONCLUSIONS}

Fractal dimension measure and post edge detection results for MR images of type PD and T2w, provided by the first- or second-order derivative filters, allow us to conclude that the fractal dimension is a proper approach for a correct classification of healthy and diseased patients. This proposed solution can be extensively used for second-order filters which provide more feasible results.

\section{REFERENCES}

1. S. Moldovanu, L. Moraru, D. Bibicu, Bio-Structural Analysis of Breast Using Fractal Analysis in Ultrasound Images, Annals of Dunărea de Jos University Fascicle II, Anul III (XXXIV), No. 1, pp. 66-72, 2011.

2. Aftab, An Overview of Image Processing, International Journal of Advnacement in Engineeing Technology, Management \& Applied Science, Volume 1, Issue 4, 2014, pp. 23 29, 2014.

3. Q. Sun , Y. Hou, Q. Tan, C. Li , M. Liu, A robust edge detection method with sub-pixel accuracy, Optik - International Journal for Light and Electron Optics, Volume 125, Issue 14, pp. 3449-3453, 2014.

4. Mohammed Mahmood, H. Hakan Maras, E. Elbaş1, Measurement of Edge Detection Algorithms in Clean and Noisy Environment, Conference: 2014 IEEE 8th International Conference on Application of Information and Communication Technologies, Ed. IEEE, Astana, Kazakhstan, 2014.

5. M. Roushdy, Comparative Study of Edge Detection Algorithms Applying on the Grayscale Noisy Image Using Morphological Filter, GVIP Journal, Volume 6, Issue 4, pp.17-23, 2006.

6. Y. Park, K. Lee, T. R. Ziegler, G. Martin, G. Hebbar, B. Vidakovic, D. P. Jones, Multifractal Analysis for Nutritional Assessment, PLOS ONE, Volume 8, Number 8, pp. e69000, 10.1371/journal.pone.0069000, 2013.

7. B. B. Mandelbrot, The Fractal Geometry of Nature (Freeman, New York, 1983), p. 113.

8. S. Majumdar and R. R. Prasad, The fractal dimension of cerebral surfaces using magnetic resonance images, Journal Computers in Physics, Volume 2, Issue 6, pp. 69-73, 1988.

9. F. J. Esteban, J. Sepulcre, J. Ruiz de Miras, J. Navas, N. Vélez de Mendizábal, J. Goñi, J. Ma Quesada, B. Bejarano, P Villoslada, Fractal dimension analysis of grey matter in multiple sclerosis, Journal of the Neurological Sciences, Volume 282, Issues 1-2, pp. 67-71, 2009. 
10. S. A. Jayasuriya, A. W. C. Liewa, N. F. Law, Brain Symmetry Plane Detection based on Fractal Analysis, Computerized Medical Imaging and Graphics, Volume 37, Issues 7-8, pp. 568-580, 2013.

11. F. T. Hoyos, R. B. Navarro, J. V. Villadiego, M. G. Martelo, Geometrical study of Astrocytomas through Fractals and Scaling analysis, Applied Radiation and Isotopes,Volume 141, pp. 250-256, 2018.

12. M. Fernández-Martínez, M. A. Sánchez-Granero, Fractal dimension for fractal structures, Topology and its Applications, Volume 163, Issue 15, pp . 93-111, 2014.

13. S. Kullback, R. A. Leibler, On information and sufficiency, Annals of Mathematical Statistics, Vol. 22, No. 1, pp. 79-86, 1951.

14. S. Moldovanu, L. Moraru, D. Stefanescu, D. Bibicu, Edge-preserving filters in a boundary options context, Annals Of "Dunarea De Jos" University of Galati Mathematics, PHYSICS, theoretical mechanics, Fascicle II, Year VIII (XXXIX), No. 1, pp. 51-57, 2017.

15. S. Kumar,M. Singh, D.K. Shaw, Comparative Analysis of Various Edge Detection Techniques in Biometric, International Journal of Engineering and Technology, Volume 8, No 6, pp. 2452-2459, 2016-2017.

16. G. T. Shrivakshan, C. Chandrasekar, A comparison of various edge detection techniques used in image processing, IJCSI International Journal of Computer Science, Volume 9, Issue 5, 2012, No 1.2012.

17. S. Cui, Y. Wang Xiaoqing Qian, Z. Deng, Image Processing Techniques in Shockwave Detection and Modeling, Journal of Signal and Information Processing, 2013, 4, pp. 109-113 doi:10.4236/jsip.2013.43B019

18. J. Wan, X. He, P. Shi, An Iris Image Quality Assessment Method Based on Laplacian of Gaussian Operation, Conference on Machine Vision Applications, May 16-18, 2007, Tokyo, JAPAN, 2007.

19. R. Lopes, N. Betrouni, Fractal and multifractal analysis: A review, Journal Medical Image Analysis, Volume 13, Issue 4, 2009, pp. 634-649, 2009.

20. J. Bhattacharya and H. Petsche, Universality in the brain while listening to music, Journal The Royal Society, Volume 268, Issue 1484, 2001, pp. 2423-2433, 2001.

21. X. Wan, W. Wang, J. Liu and T. Tong, Estimating the sample mean and standard deviation from the sample size, median, range and/or interquartile range, Journal BMC Medical Research Methodology, 19 December 2014, https://doi.org/10.1186/1471-2288-14-135. 